\title{
A MODIFIED APPROACH FOR CHANGE DETECTION USING CHANGE VECTOR ANALYSIS IN POSTERIOR PROBABILITY SPACE
}

\author{
S. A. Azzouzi ${ }^{\mathrm{a}, \mathrm{b},},{ }^{*}$ A. Vidal $^{\mathrm{a}}$, H. A. Bentounes ${ }^{\mathrm{b}}$ \\ ${ }^{\text {a } I n s t i t u t o ~ d e ~ T e l e c o m u n i c a c i o n e s ~ y ~ A p l i c a c i o n e s ~ M u l t i m e d i a ~(i T E A M), ~ U n i v e r s i t a t ~ P o l i t e ̀ c n i c a ~ d e ~ V a l e ̀ n c i a, ~}$ \\ Valencia, Spain - azzouzi.soufiane@hotmail.com, avidal@dcom.upv.es \\ ${ }^{\mathrm{b}}$ Component, signal and systems laboratory, University Abdelhamid Ibn Badis of Mostaganem, \\ Mostaganem, Algeria - bhadjadda@gmail.com
}

KEY WORDS: Modified Change Vector Analysis Posterior probability Space (MCVAPS), Classification, Spatiotemporal change, Land Cover/Land Change, Landsat-TM data, Landsat-ETM data.

\begin{abstract}
:
The multispectral and multitemporal data coming from satellites allow us to extract valuable spatiotemporal change. Consequently, Earth surface change detection analysis has been used in the past to monitor land cover changes caused by different reasons. Several techniques have been used for that purpose and change vector analysis (CVA) has been frequently employed to carry out automatic spatiotemporal information extraction. This work describes a modified methodology based on Supervised Change Vector Analysis in Posterior probability Space (SCVAPS) with the final aim of obtaining a change detection map in Blida, Algeria. The proposed technique is a Modified version of Supervised Change Vector Analysis Posterior probability Space (MSCVAPS) and it is applied at the same region that the original technique studied in the literature. The classical Maximum Likelihood classifier is the selected method for supervised classification since it provides good properties in the posterior probability map. An improved method for threshold determination based on Double Flexible Pace Search (DFPS) is proposed in this work and it is employed to obtain the most adequate threshold value. Then, the MSCVAPS approach is evaluated by two cases study of the land cover change detection in the region of Blida, Algeria, and in the region of Shunyi District, Beijing, China, using a pair of Landsat Thematic Mapper images and pair of Landsat Enhanced Thematic Mapper images, respectively. The final evaluation is given by the overall accuracy of changed and unchanged pixels and the kappa coefficient. The results show that the modified approach gives excellent results using the same area of study that was selected in the literature.
\end{abstract}

\section{INTRODUCTION}

Advances in satellite image acquisition and new storage technologies have led to a huge amount of information in the spatiotemporal domain. These data can be used and analyzed to produce land cover detection and land cover change information. Change detection methods are based on processing spatiotemporal data in a convenient way in order to access the change information as described in (Armenakis et al., 2002), (Barnes et al., 2007), (Tang et al., 2007) and (Umamaheshwaran et al., 2007). The detection of possible differences that may occur over time is a key factor when analyzing the state of an area. These differences may be caused by several phenomena, that include human activity and natural causes, as described in (Nori et al., 2009) and (Singh, 1989).

The technique of Change Vector analysis (CVA) has been vastly used in the past decade for change detection in various studies such as (Bovolo and Bruzzone, 2007), (Malila, 1980) and (Xian. et al., 2009). Many change detection techniques were summarized by (Lu et al., 2004) as algebraic algorithm, advanced techniques, visual analysis and others. Each technique provided its own features and that study also gave an effective guidance to design rules for modifying and developing CVA (Singh, 1989). Among all techniques, change vector analysis is able to describe the output data in terms of total magnitude of change and angle of direction between two different time samples from multispectral satellite images as described in (Collins and Woodcock, 1994), (Jensen, 1995) and (Johnson and Kasischke, 1998).

${ }^{*}$ Corresponding author. This is useful to know for communication with the appropriate person in cases with more than one author
However, some shortcomings are still not solved in change detection techniques, such as classification error accumulation. A CVA method called Modified Supervised Change Vector Analysis Posterior probability Space (MSCVAPS) based on the technique presented in (Chen et al., 2011) is carried out in this work. It has been applied to detect land cover change obtaining excellent results in the studied areas when compared to the literature results. This developed method includes two steps, the first step implements the Double window Flexible Pace Search (DFPS) algorithm to generate a binary "change" or "no-change" image and the second step uses the scalar product to obtain the classified change map.

In order to assess the effectiveness of MSCVAPS, the technique has been evaluated into two study areas : (1) Shunyi District, Beijing, China, (2) Blida, Algeria. One of them (China) is chosen for comparison purposes because it was the area selected for analysis in the literature (Chen et al., 2011). The second test area (Algeria) is the real object of study, since an operative tool to detect change has been demanded in the area of Blida in Algeria. The final quality is given by the Kappa coefficient and the overall accuracy of changed unchanged pixels like the methods presented in (Cha, 2007), (Chen et al., 2003), (Chen et al., 2011), (Jensen, 1995) and (Nackaerts et al., 2005). A study has chosen the same China region used in (Chen et al., 2011) for comparison purposes.

This work describes a new modified method for the assessment of CVA techniques. It also applies this methodology to two different areas for comparison purposes. 


\begin{tabular}{cccccccc}
\hline Dates & Landsat ID N $^{\circ}$ & Instrument & Path/Row & LPGS version & Data type & Solar azimuth & Solar elevation \\
\hline $30-04-2000$ & ETM 7 & SAM & $123 / 032$ & 12.3 .1 & L1T & 137.97 & 58.50 \\
$10-04-2010$ & ETM 7 & Bumper & $123 / 032$ & 12.5 .0 & L1T & 142.46 & 51.94 \\
\hline
\end{tabular}

Table 1: Characteristics of the Landsat 7 images in the Chinese region.

\begin{tabular}{cccccccc}
\hline Dates & Landsat ID N & Instrument & Path/Row & LPGS version & Data type & Solar azimuth & Solar elevation \\
\hline 14-06-2002 & TM 5 & Bumper & $196 / 035$ & 12.5 .0 & L1T & 111.30 & 63.40 \\
04-06-2010 & TM 5 & Bumper & $196 / 035$ & 12.5 .0 & L1T & 118.23 & 65.98 \\
\hline
\end{tabular}

Table 2: Characteristics of the Landsat 5 images in the Algerian region.

\section{STUDY AREA DESCRIPTION}

\subsection{Area selection}

The images of the selected study areas have been acquired from the Landsat program by different sensors, and they are freely available in the United States Geological Survey (USGS) archive in (USGS, 2014). The first study area images were acquired by Landsat-7 ETM (Enhanced Thematic Mapper) sensor. All bands, except from the thermal infrared one, were used in the methodology. The chosen area is placed in Shunyi District, Beijing, China, which lies from latitude $40.20^{\circ}$ (North) to $40.04^{\circ}$ (North) and longitude $116.68^{\circ}$ (East) to $116.53^{\circ}$ (East). The image characteristics and acquisition dates are shown at Table 1 . The selected images were obtained at the same month to reduce seasonal variance. A sub-area with 560x442 pixels was finally selected for further study. It covers a total area equal to $223.67 \mathrm{~km}^{2}$. This test area was chosen for validation purposes because it had been used in the literature to present the original CVA methodology in (Chen et al., 2011). Fig. 1 shows the general map and the subarea location.

The second data set used in this work consisted of two spatiotemporal images acquired by Landsat-5 TM (Thematic Mapper) sensor in the region of Blida, Algeria. All bands, except from the thermal infrared one, were used in the methodology. The change detection analysis in this area was the main objective of this work. It presents a total surface equal to $788.18 \mathrm{~km}^{2}$, located at a latitude of $36.34^{\circ}$ (North) to $36.13^{\circ}$ (North) and longitude of $1.98^{\circ}$ (East) to $2.36^{\circ}$ (East). In the study, a sub-area of $1130 \times 775$ pixels was chosen and it is shown in Fig. 2 and Fig.3. Table 2 presents more information about the data set in Algeria and their acquisition characteristics.

\section{METHODOLOGY}

\subsection{Image pre-processing}

The selected images may contain pixel spectral changes caused by several factors, such as soil moisture, atmospheric conditions, sun angle variation, sensor type and other parameters, apart from the real change detection cause. These pixel spectral changes must be removed to make that the changes are only caused by surface changes. A very important factor in change detection is to make sure that the location of the same surface features is coherent. If the accuracy of image co-registration is low, a high amount of pixels will be detected as changed just because of image shifting.

Therefore, pre-processing of the satellite imagery includes radiometric calibration, atmospheric reflectance, atmospheric normalization, geometric correction, image registration, and image rectification. The radiometric correction is critically needed due to several factors like changing sensor type parameters, different solar azimuth and solar zenith angles, different atmospheric conditions for scattering and absorption (Yan. and Shaker, 2014). In

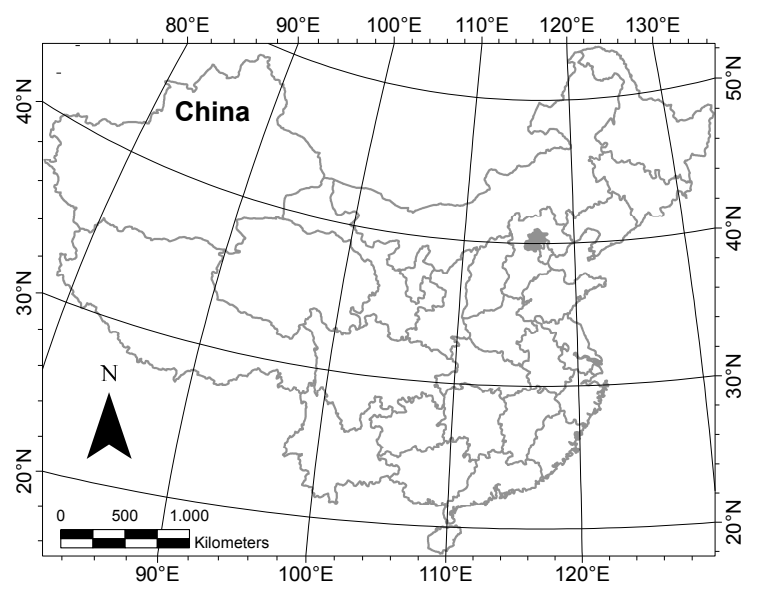

(a)

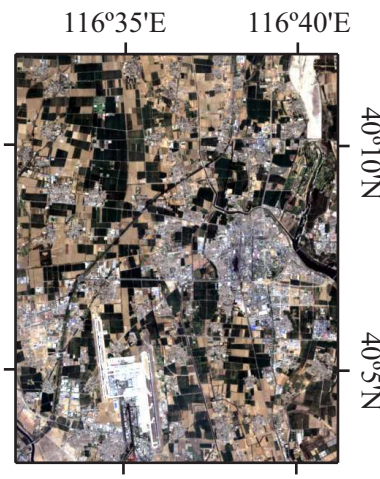

(b)

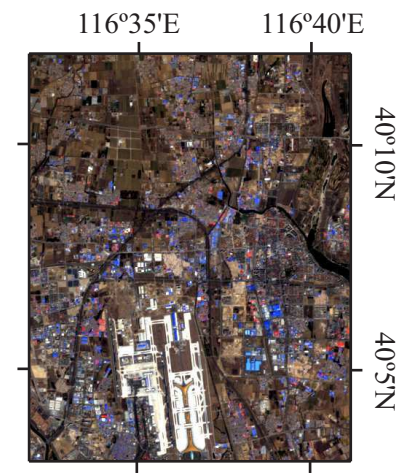

(c)
Figure 1: The studied area of China: Map location of the subarea in a), satellite image acquired on April 30, 2000 in b), and satellite image acquired on April 10, 2010 in c).

this work, atmospheric correction has been carried out using the dark substraction algorithm and radiometric correction has been done using the Landsat Correction algorithm of the ENVI software. The geometric correction is also applied in order to resemble the truth ground location coordinates following (Xiangyang. et al., 2010). Then, image registration and image rectification finally provide the corrected image that will be used for the analysis (Reji and Vidya, 2012).

After geometric rectification and image-to-image registration provided by the ENVI tools using 100 control points, the Root Mean Square Error (RMSE) between both images were computed and they were approximately equal to 0.21 , and 0.11 pixels for the sub-areas represented in the Figure.1, 3, respectively.

\subsection{Change Vector Analysis}

Change Vector Analysis is a change detection methodology that keeps interesting information from classified images and enables further interpretation than other change detection methods, as explained in in (Malila, 1980) and (Michalek et al., 1993). The 


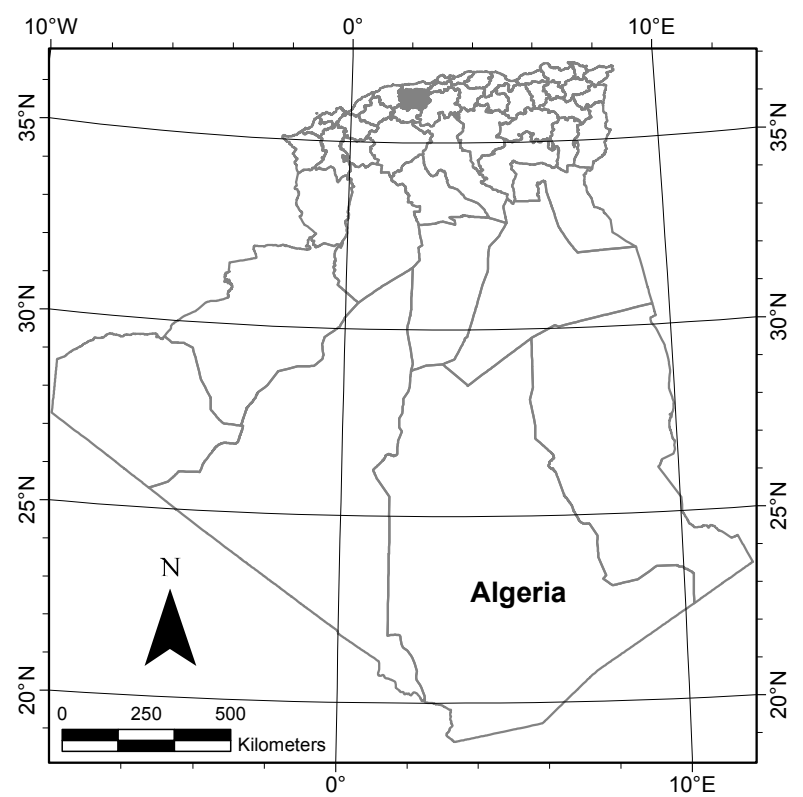

Figure 2: Map localisation of the studied area in Algeria.

global idea of CVA consists in computing the spectral change vectors by the subtraction of pairs of spectral bands. A change vector is described by the magnitude of change and the angle of change between two images obtained at the moment $t_{1}$ and $t_{2}(\mathrm{Lu}$ et al., 2004). When a threshold is applied to this change vector based on their magnitude, then a possible change is determined, as described in (Chen et al., 2003) and (Nackaerts et al., 2005).

The Modified Supervised Change Vector Analysis Posterior probability Space (MSCVAPS) described in (Chen et al., 2011) has been taken as the initial point for this study. The first step in the methodology is to carry out a supervised classification that provides a posterior probability map for each scene. In this work, 5 classes were used in the classification process: bare soil, dense vegetation, no-dense vegetation, urban and water areas. In our case, the Maximum Likelihood Classifier (MLC) was the chosen classification technique using the spectral bands $b_{1}, b_{2}, b_{3}, b_{4}, b_{5}$ and $b_{7}$ of the Landsat TM or ETM instrument, respectively. The supervised MLC classifier requires a training process that needs ground truth data. Since no in situ information was available, visual information was obtained from the satellite images. The total amount of training pixels used in the classification process were $10.74 \%$ of the total sub-area in China and $6.02 \%$ of the total sub-area in Algeria.

The Maximum Likelihood classifier provides the posterior probability vectors in the space-time domain:

$$
\begin{gathered}
H=\left(h_{1}, h_{2}, \ldots, h_{n}\right), \\
G=\left(g_{1}, g_{2}, \ldots, g_{n}\right),
\end{gathered}
$$

where $H$, and $G$ represents the posterior probability vector at the time $t_{1}$ and $t_{2}$, respectively. The index $1,2, \ldots, n$ refers to each one of the classes consequently defined.

The change vector of the posterior probability space can be defined as:

$$
\Delta M=G-H,
$$

where $\Delta M$ is the posterior probability difference map for each pixel, and it also contains the information of the change that has taken place between the two images. The magnitude of change

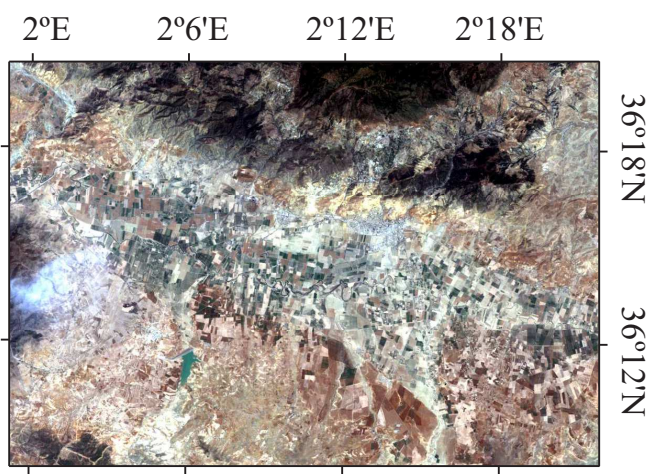

(a)

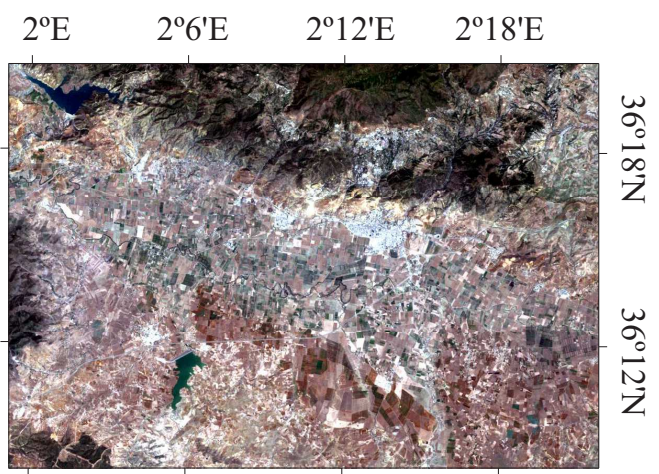

(b)

Figure 3: The studied area of Algeria: image acquired on June 14, 2002 in a), and image acquired on June 04, 2010 in b).

vector is given by the following expression:

$$
\|\Delta M\|=\left(\sum_{i=1}^{n}\left(G_{i}-H_{i}\right)^{2}\right)^{1 / 2} .
$$

In this context, a large value for $\|\Delta M\|$ indicates a strong possibility of a changed pixel.

\subsection{Threshold determination}

The stage of the threshold selection is one of the most important steps in the procedure to determine whether a pixel is changed or not. A supervised threshold determination method using the Double Flexible Pace Search (DFPS) algorithm, described in (Chen et al., 2003), was employed in this work.

\subsection{Change type discrimination}

The change type discrimination is used to identify the considered changed pixels in the Land Use/Land Cover (LULC) map. It is obtained by the analysis of the posterior probability map and the pixel-wise radiometric comparison as described in the literature in (Chen et al., 2011).

The change class information between the space-time $t_{1}$ and $t_{2}$ can be obtained from the direction of $\Delta M$. A pure pixel transformed from class $a$ to other class $b$ during a period of time would present the following change vector

$$
\begin{gathered}
\Delta P_{a b}=P_{b}-P_{a}, \\
P_{z}=\left(p_{z 1}, p_{z 2}, \ldots, p_{z n}\right), \\
p_{z i}= \begin{cases}p_{z i}=1, & \text { if } i=z \\
p_{z i}=0, & \text { if } i \neq z\end{cases}
\end{gathered}
$$


where $P_{z}$ describes the posterior probability vector of a pure pixel of class $\mathrm{z}$. When the angle between the actual vector $\Delta M$ and the theoretical vector $\Delta P_{a b}$ is small, then the corresponding pixel has probably changed from class $a$ to class $b$. Then, the change type can be easily determined from the scalar product, searching for the maximum value for the scalar product from all possible combinations of pairs $a-b$ :

$$
\Delta M \cdot \Delta P_{a b}=\sum_{i=1}^{n} \Delta M_{i} \cdot\left(\Delta P_{a b}\right)_{i} .
$$

\section{RESULTS}

The presented methodology has been applied to the selected data sets corresponding to the sub-area in China and Algeria.

The posterior probability vectors $H$ for time $t_{1}$ and $G$ for time $t_{2}$ are shown in Fig. 4 and Fig. 5 for the sub-areas of China and Algeria respectively. These are results from the MLC classifier using $b_{1}, b_{2}, b_{3}, b_{4}, b_{5}$ and $b_{7}$ bands. A previously described, 5 classes were used for classification: bare soil, dense vegetation, no-dense vegetation, urban and water areas. This limited range of classes allow the methodology to get a very accurate classification map even with a low amount of training pixels. The total amount of training pixels used in the classification process were $10.74 \%$ of the total sub-area in China and $6.02 \%$ of the total sub-area in Algeria. An important amount of changed pixels is clearly visible across the China scenes. The Algerian scenes show change in vegetation and they also show differences in valuable water bodies.

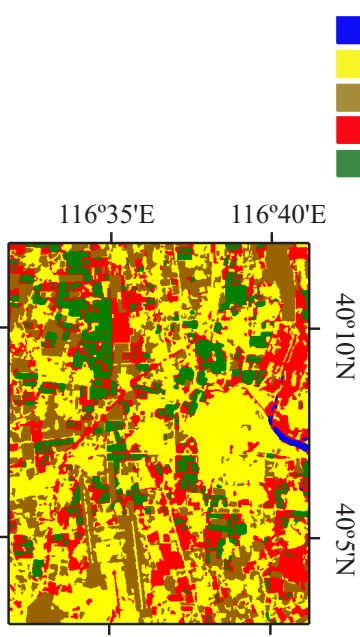

a)

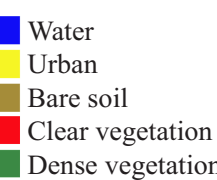

Dense vegetation
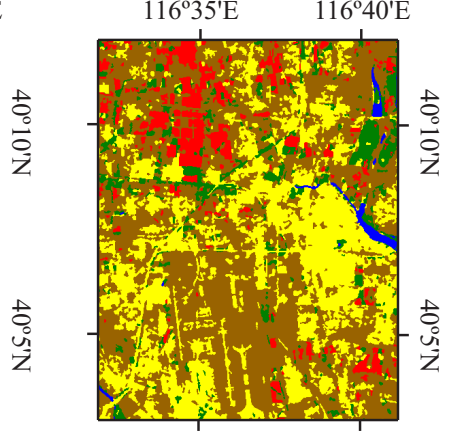

b)
Figure 4: The posterior probability map for the China region: a) acquired on April 30, 2000, and b) acquired on April 10, 2010.

In order to evaluate the performance of the proposed MSCVAPS method, the overall accuracy and kappa coefficient obtained in determining changed or unchanged pixels were computed using the MSCVAPS results and a reference data set. This validation data set used was obtained again from visual inspection and it was different from the training data set, in order to get a fair accuracy assessment.

The changed/unchanged confusion matrix of MSCVAPS at the region of China is shown in Table 3. It represents the results of 247520 sample pixels. The reference validation data set in China was equal to $13.6 \%$ of the total sub-area. A remarkable Kappa coefficient of 0.827 and an overall accuracy of $91.50 \%$ were achieved by the proposed method. This change detection

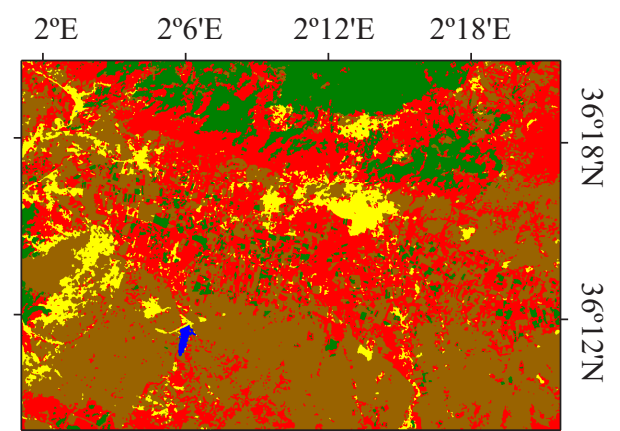

b)
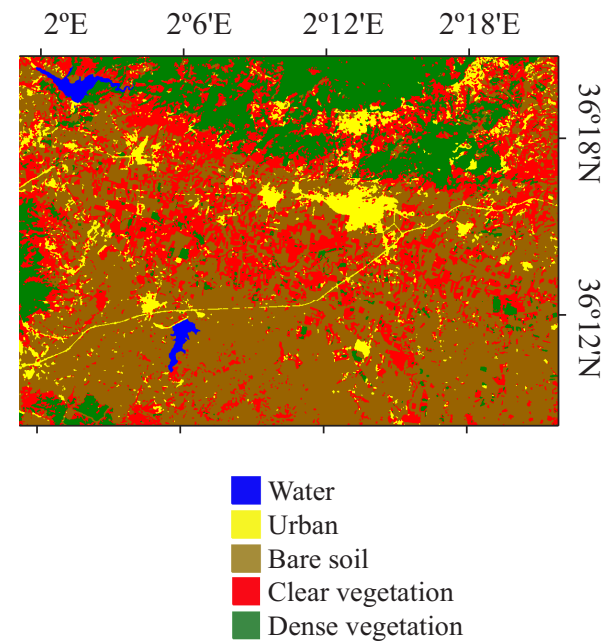

Figure 5: The posterior probability map for the Blida region: acquired on June 14, 2002 in a), and acquired on June 04, 2010 in b).

result was compared with the results presented in the literature in (Chen et al., 2011). The comparison indicates that the proposed method gives excellent results. Therefore, the method is validated for its use in other regions.

Then, once the methodology is validated, Table 4 shows the confusion matrix of changed/unchanged pixels at the sub-area of Algeria, that contains 875750 pixels. The reference validation data set was equal to $13.6 \%$ of the total sub-area. The obtained Kappa coefficient was equal to 0.865 and the overall accuracy was equal to $93.96 \%$.

These results show the effectiveness of the change detection methodology in the selected regions using Landsat data and the defined classes. Kappa coefficient is above 0.7 and the omission and commission errors of the changed and unchanged pixels were good balanced in both test areas.

\begin{tabular}{|c|c|c|c|c|c|}
\hline \multirow{2}{*}{\multicolumn{2}{|c|}{ Number of pixels }} & \multicolumn{4}{|c|}{ Reference changed } \\
\hline & & UP & $\mathrm{CP}$ & Sum & $\mathrm{CE}$ \\
\hline \multirow{4}{*}{$\begin{array}{l}\text { Classified } \\
\text { changed }\end{array}$} & UP & 100008 & 9348 & 109356 & $8.55 \%$ \\
\hline & $\mathrm{CP}$ & 11713 & 126451 & 138164 & $8.48 \%$ \\
\hline & Sum & 111721 & 135799 & 247520 & \\
\hline & $\mathrm{OE}$ & $10.48 \%$ & $6.88 \%$ & & \\
\hline
\end{tabular}

Overall accuracy $91.50 \%$, Kappa coefficient 0.827

NB: UP, CP, CE, OE represent the unchanged pixels, changed

pixels, commission error, and omission error respectively.

Table 3: Changed/unchanged confusion matrix for the sub-area in China.

The total change maps obtained with the MCVAPS algorithm are 


\begin{tabular}{lccccc}
\hline \multirow{2}{*}{ Number of pixels } & \multicolumn{4}{c}{ Reference changed } \\
\cline { 3 - 6 } & & UP & CP & Sum & CE \\
\hline \multirow{3}{*}{ Classified } & UP & 554799 & 23075 & 577874 & $3.99 \%$ \\
changed & 29801 & 268075 & 297876 & $10.00 \%$ \\
& Sum & 584600 & 291150 & 875750 & \\
& OE & $5.10 \%$ & $7.93 \%$ & &
\end{tabular}

Overall accuracy $93.96 \%$, Kappa coefficient 0.865

NB: UP, CP, CE, OE represent the unchanged pixels, changed pixels, commission error, and omission error respectively.

Table 4: Changed/unchanged confusion matrix for the sub-area in Algeria.

shown in Fig. 6 and Fig. 7 for the Chinese region and the Algerian region, respectively. In the case of China, it can be demonstrated that many pixels appear as changed due to urban growth and change of land use. This zone is very dynamic and this effect could be easily assessed by the use of the proposed methodology.

In the area of Algeria, a large number of pixels were also labeled as changed due to human activity and different climatic conditions. The forest areas were increased by $3.18 \%$, the urban areas were increased by $2.9 \%$ and water areas were also increased by $0.62 \%$. On the contrary, grassland and the bare soil areas were decreased by $3.64 \%$ and $3.06 \%$, respectively. The urban changes were mainly caused by new building construction. The changes in forest areas were caused by the plantation of new trees in mountainous areas and this fact is also related to the reduction of the grassland area.

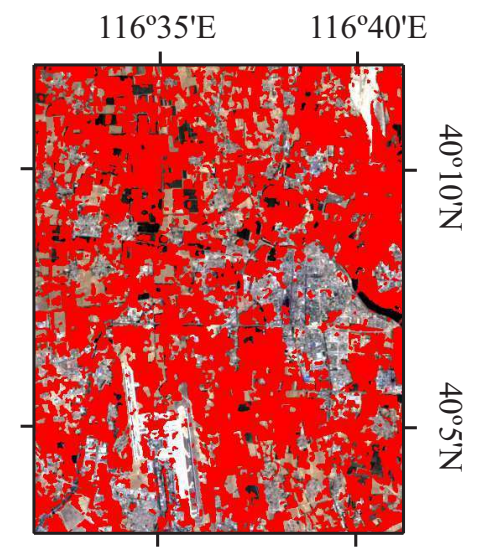

Figure 6: Pixels in red represent the changed areas detected by MSCVAPS method in the sub-area of China.

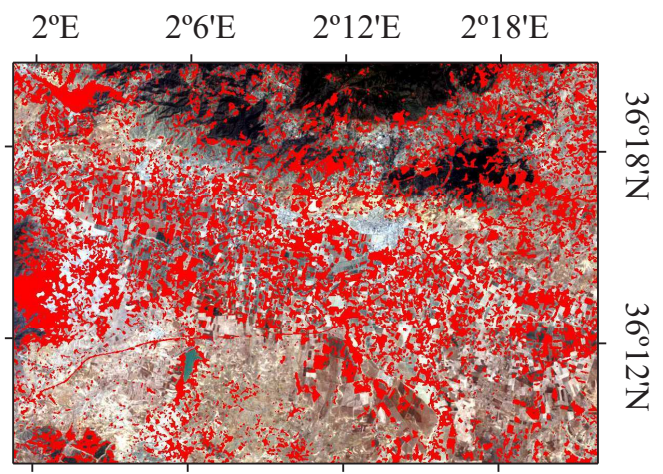

Figure 7: Pixels in red represent the changed areas detected by MSCVAPS method in the sub-area of Algeria.

\section{DISCUSSION AND CONCLUSION}

This work has described a methodology based on the existing change vector analysis in the posterior probability space and it has been applied in two different scenarios. The performance of the proposed algorithm has been evaluated with validation data obtained by visual inspection. The accuracy evaluation provides a Kappa coefficient greater than 0.7 for both test areas. The results suggest that the MCVAPS is effective and it has a strong potential for Remote Sensing change detection methods.

The quality of the data plays a very important role, as acquisition of data should be made at different years choosing the same season. Besides, image registration has a strong impact on the final change detection methodology because it can introduce severe artifacts due to pixel location mismatch. In this work, excellent results are obtained thanks to the good quality of the satellite images, the strict radiometric and geometric correction and the accurate co-registration given by the proposed methodology.

In general, it is difficult to collect global information for all years of land use/land cover change information. The presented methodology takes advantage of the spatiotemporal differences between two images acquired at different dates. For that reason, CVAPS is an effective approach in this scenario. Further development of the CVA technique is expected in the future, with an enhancement of robust and automatic algorithms for land use/land cover detection.

\section{ACKNOWLEDGEMENTS}

This research has been done in the frame of EMMAG funding.

\section{REFERENCES}

Armenakis, C., Cyr, I. and Papanikolaou, E., 2002. Change detection methods for revision of topographic databases. In: Symposium on Geospatial Theory, Processing and Applications., ISPRS.

Barnes, C., Fritz, H. and Yoo, J., 2007. Hurricane disaster assessments with image-driven data mining in high-resolution satellite imagery. Geoscience and Remote Sensing, IEEE Transactions on 45(6), pp. 1631-1640.

Bovolo, F. and Bruzzone, L., 2007. A theoretical framework for unsupervised change detection based on change vector analysis in the polar domain. Geoscience and Remote Sensing, IEEE Transactions on 45(1), pp. 218-236.

Cha, S., 2007. Comprehensive survey on distance/similarity measures between probability density functions. In: International Journal of Mathematical Models and Methods in Applied Sciences, Vol. 1, IEEE, pp. 300-307.

Chen, J., Chen, X., Cui, X. and Chen, J., 2011. Change vector analysis in posterior probability space: A new technique for land cover change detection. In: IEEE Geo-science and Remote Sensing Letters, Vol. 8, IEEE, pp. 317-321.

Chen, J., Gong, P., He, C., Pu, R. and Shi, P., 2003. Landuse/land-cover change detection using improved change-vector analysis. In: Photogrammetric Engineering and Remote Sensing, Vol. 69, ASPRS, pp. 369-379.

Collins, J. and Woodcock, C., 1994. Change detection using the Gramm-Schmidt transformation applied to mapping forest mortality. Remote Sensing of Environment 50(3), pp. 267-279. 
Jensen, J. R., 1995. Introductory Digital Image Processing: A Remote Sensing Perspective. 2 edn, Prentice Hall PTR, Upper Saddle River, NJ, USA.

Johnson, R. D. and Kasischke, E. S., 1998. Change vector analysis: A technique for the multispectral monitoring of land cover and condition. International Journal of Remote Sensing 19(3), pp. 411-426.

Lu, D., Mausel, P., Brondzio, E. and Moran, E., 2004. Change detection techniques. International Journal of Remote Sensing 25(12), pp. 2365-2401.

Malila, W., 1980. Change vector analysis: An approach for detecting forest changes with landsat. In: Proceedings of Sixth Annual Symposium on Machine Processing of Remotely Sensed Data: Soil Information Systems and Remote Sensing and Soil Survey, IEEE, pp. 326-335.

Michalek, J. L., Wagner, T. W., Luczkovich, J. J. and Stoffle, R. W., 1993. Multispectral change vector analysis for monitoring coastal marine environments. Photogrammetric Engineering \& Remote Sensing 59(3), pp. 381-384.

Nackaerts, K., Vaesen, K., Muys, B. and Coppin, P., 2005. Comparative performance of a modied change vector analysis in forest change detection. In: Int. Journal of Remote Sensing., Vol. 26, Taylor \& Francis, pp. 839-852.

Nori, W., Sulieman, H. and Niemeyer, I., 2009. Detection of land cover changes in el rawashda forest, sudan: A systematic comparison. In: Geoscience and Remote Sensing Symposium,2009 IEEE International,IGARSS 2009, Vol. 1, pp. I-88-I-91.

Reji, R. and Vidya, R., 2012. Comparative analysis in satellite image registration. In: Computational Intelligence Computing Research (ICCIC), 2012 IEEE International Conference on, pp. 1-6.

Singh, A., 1989. Review article digital change detection techniques using remotely-sensed data. International Journal of Remote Sensing 10(6), pp. 989-1003.

Tang, J., Wang, L. and Yao, Z., 2007. Spatiotemporal urban landscape change analysis using the markov chain model and a modified genetic algorithm. International Journal of Remote Sensing 28(15), pp. 3255-3271.

Umamaheshwaran, R., Bijker, W. and Stein, A., 2007. Image mining for modeling of forest fires from meteosat images. Geoscience and Remote Sensing, IEEE Transactions on 45(1), pp. 246-253.

USGS, 2014. GLOVIS http://glovis.usgs.gov/.

Xian., G., Homer., C. and Fry, J., 2009. Updating the 2001 national land cover database land cover classification to 2006 by using landsat imagery change detection methods. Remote Sensing of Environment 113(6), pp. 1133-1147.

Xiangyang., S., Conggui., L. and Yizhen, S., 2010. Comparison and analysis research on geometric correction of remote sensing images. In: Image Analysis and Signal Processing (IASP), 2010 International Conference on, pp. 169-175.

Yan., W. Y. and Shaker, A., 2014. Radiometric correction and normalization of airborne lidar intensity data for improving landcover classification. Geoscience and Remote Sensing, IEEE Transactions on 52(12), pp. 7658-7673. 